\title{
Gilteritinib improves outcomes in AML
}

Patients with acute myeloid leukaemia (AML) who fail to respond to, or relapse after chemotherapy often have dismal outcomes. Now, data from the phase III ADMIRAL trial confirm the efficacy of the FLT3 inhibitor gilteritinib in patients with activating FLT3 mutations in this setting.

A total of 371 adult patients with FLT3mutant AML who either failed to respond to, or had disease relapse following complete remission in response to anthracycline-based induction chemotherapy were randomized (2:1) to receive gilteritinib or investigator's choice of salvage chemotherapy. The primary end points were overall survival (OS) and percentage of patients with complete remission and full or partial haematological recovery.

After a median follow-up duration of 17.8 months, patients in the gilteritinib group had a median OS of 9.3 months versus 5.6 months in the chemotherapy group (HR 0.64, 95\% CI 0.49-0.83; $P<0.001$ ). Furthermore, $34 \%$ of patients receiving gilteritinib had complete remission with at least partial haematological recovery versus $15.3 \%$ in the chemotherapy group (risk difference 18.6\%, 95\% CI 9.8-27.4), including complete haematological recovery rates of $21.1 \%$ and $10.5 \%$, respectively.

Common treatment-related grade $\geq 3$ adverse events among patients receiving gilteritinib included febrile neutropenia (45.9\%), anaemia (40.7\%), and thrombocytopenia (22.8\%). Patients receiving gilteritinib were more likely to discontinue treatment owing to adverse events than those receiving chemotherapy (15\% versus $7.3 \%)$ and had a similar risk of treatment-emergent adverse events leading to death $(5.7 \%$ versus $7.3 \%)$. However, when adjusted for the duration of treatment exposure, patients in the gilteritinib arm had fewer adverse events than those in the chemotherapy arm (19.34 versus 42.44 events per patient-year).

These findings confirm the superior efficacy of gilteritinib versus chemotherapy for patients with FLT3-mutant AML.

Peter Sidaway

ORIGINAL ARTICLE Perl, A. E. et al. Gilteritinib or chemotherapy for relapsed or refractory FLT3-mutated AML. N. Engl. J. Med. 381, 1728-1740 (2019)

\section{Sequence of AR inhibitors affects outcome}

The availability of CYP17A1 inhibitors (such as abiraterone) and androgen receptor (AR) antagonists (such as enzalutamide) has improved the outcomes of men with metastatic castration-resistant prostate cancer (mCRPC). However, the optimal sequence of agents is unclear and resistance mechanisms are likely to overlap. Now, data from a phase II trial indicate that abiraterone should be administered prior to enzalutamide in this setting.

A total of 202 men with newly diagnosed $\mathrm{mCRPC}$ were randomly assigned $(1: 1)$ to receive abiraterone plus prednisone, followed by enzalutamide on biochemically confirmed disease progression (group A), or vice versa (group B). Time to second biochemical progression, as indicated by serum prostate-specific antigen (PSA) level, was the primary end point.

At the latest cut-off, similar numbers of patients had crossed over in both arms $(72 \%$ in group $\mathrm{A}$ and $74 \%$ in group B). Patients in group A had a significantly longer median time to second progression than those in group B (19.3 months versus 15.2 months, (HR $0.66,95 \% \mathrm{Cl} 0.45-0.97$; $P=0.036$ ). This improvement was supported by a superior second-line biochemical response rate $(36 \%$ versus $4 \% ; P<0.0001)$ and improved median time to progression on second-line therapy (3.5 months versus 1.7 months, HR 0.42, 95\% Cl 0.28-0.65; $P<0.0001$ ), respectively. No significant difference was observed in median time to progression on first-line therapy.

Adverse events were similar to those observed in other trials involving these agents. Hypertension ( $18 \%$ versus $15 \%$ ) and fatigue ( $5 \%$ versus $3 \%$ ) in group $A$ versus group $B$, respectively, were the most frequent grade 3-4 adverse events in response to second-line therapy.

These data demonstrate the superiority of abiraterone followed by enzalutamide for men with newly diagnosed mCRPC; however, how these data relate to the castrationsensitive setting, in which abiraterone is now approved, and to other agents of the same class, remains unclear.

Peter Sidaway

ORIGINAL ARTICLE Khalaf, D. J. et al. Lancet Oncol. https://doi.org/10.1016/S1470-2045(19)30688-6 (2019)

\section{PD-1 inhibition} enters the frontline

In 2016, the FDA approved PD-1 inhibition with either pembrolizumab or nivolumab for the second-line treatment of patients with recurrent and/or metastatic head and neck squamous cell carcinoma (HNSCC), after progression on platinum-based chemotherapy. Now, data from KEYNOTE-048 demonstrate the efficacy of pembrolizumab in the frontline.

In this three-arm phase III trial, 882 patients received either standard-of-care (SoC) cetuximab plus chemotherapy (carboplatin or cisplatin and 5-fluorouracil), pembrolizumab alone or pembrolizumab plus chemotherapy. Patients were enrolled irrespective of tumour PD-L1 expression, but were stratified by PD-L1 combined positive score (CPS; $\geq 1 \%$ or $\geq 20 \%$ ).

In the intention-to-treat population, pembrolizumab plus chemotherapy improved overall survival (OS) compared with SoC treatment (13.0 months versus 10.7 months; HR 0.77, 95\% Cl 0.63-0.93; $P=0.0034$ ), and pembrolizumab monotherapy was non-inferior to the SoC regimen (median 11.6 months versus 10.7 months; HR 0.85 , 95\% Cl 0.71-1.03). Pembrolizumab, with or without chemotherapy, improved OS in the CPS $\geq 1 \%$ and $\geq 20 \%$ subgroups, with hazard ratios of $0.60-0.78$ and $P$ values of $<0.009$; however, neither of these treatments improved progression-free survival. Interestingly, the median duration of response was $\sim 23$ months with pembrolizumab alone, regardless of CPS, compared with $<7.1$ months with this agent plus chemotherapy and $\leq 4.5$ months with the $\mathrm{SoC}$ regimen. The impressive durability of responses in the monotherapy arm was offset by a considerably lower response rate ( $17 \%$ versus $36 \%$ in both chemotherapy arms).

These data supported two new FDA approvals of pembrolizumab for the frontline treatment of patients with recurrent and/or metastatic HNSCC, in combination with chemotherapy for all patients and as monotherapy for those with a CPS $\geq 1 \%$. Notably, pembrolizumab monotherapy, the safest treatment (grade $\geq 3$ adverse event rate $55 \%$ versus $83-85 \%$ with the chemotherapy combinations), might result in durable responses in $\sim 20 \%$ of patients with PD-L1-positive HNSCC, although better biomarkers are needed to distinguish these patients from those who require additional chemotherapy.

David Killock 